\title{
Imperfect Trapping in a Random Walk with Both Species Mobile
}

\author{
Natalia C. Bustos1, Miguel A. Ré1,2 \\ ${ }^{1}$ Fa.M.A.F.-U.N.C., Córdoba, Argentina \\ ${ }^{2}$ CIII-F.R.C.-U.T.N., Córdoba, Argentina \\ Email: mgl.re33@gmail.com, re@famaf.unc.edu.ar
}

How to cite this paper: Bustos, N.C. and Ré, M.A. (2018) Imperfect Trapping in a Random Walk with Both Species Mobile. Advances in Pure Mathematics, 8, 141-153. https://doi.org/10.4236/apm.2018.81007

Received: December 21, 2017

Accepted: January 28, 2018

Published: January 31, 2018

Copyright (c) 2018 by authors and Scientific Research Publishing Inc. This work is licensed under the Creative Commons Attribution International License (CC BY 4.0).

http://creativecommons.org/licenses/by/4.0/

\begin{abstract}
It is presented here a continuous time random walk model for diffusion mediated reactions with both species mobile. The random walk is carried out over an infinite homogeneouos lattice. They are calculated the probability density for the time of reaction of a pair, the reaction rate and the time evolution of the concentration of the majority species. Analytical results are obtained in the Fourier-Laplace transform representation. Known results for a fixed trap are reobtained with appropriate marginal probabilities. It is thus justified Smoluchowski's original approximation considering the trap at a fixed position and the majority species diffusing with a coefficient sum of the individual coefficients. The results obtained are illustrated by a one dimensional model with bias.
\end{abstract}

\section{Keywords}

Mobile Trap, Diffusion Mediated Reactions, Continuouos Time Random Walk, Trapping Models

\section{Introduction}

The dynamics of diffusion mediated reactions processes has been extensively studied [1] for many years. It was the objective of these studies the proposal of theoretical models for describing chemical reactions. The schema so developed has also shown useful for the description of diverse phenomena in physics, biology or echology [2] [3] [4] [5]. The reaction process is modeled as a trapping reaction $A+T \rightarrow T$ or $A+T \rightarrow C$ and it is said diffusion controlled when the reactives have to diffuse to meet in space and then react.

One hundred years ago, Smoluchowski [6] proposed a simplified model assuming a very dilute species (the minority species denoted here as $T$, the trap) 
so that it can be considered as a single isolated spherical particle $T$ sorrounded by a swarm of diffusing pointlike particles $A$. In Smoluchowski's model particles $A$ diffusion coeficient is $D^{\prime}=D_{A}+D_{T}$ the sum of the individual coeficients of $A$ 's and $T$ 's respectively. In the original model the trapping is inmediate (perfect trapping) upon the collision of an $A$ particle with the sphere $T$. The reaction rate is computed from the flux of $A$ 's into the sphere.

Since this pioneering work different extensions have been proposed to include diffusion in disordered media [7] [8] [9] or to give a better description for the short time behaviour introducing a finite reaction time [10] (imperfect trapping). In imperfect trapping the particles may even separate after en encounter without reacting [11].

The discrete formulation of the problem is also of interest in many physical problems [12] [13] and trapping models have also been formulated based on Random Walks on lattices. Among these models we mention imperfect trapping [14] [15] with a finite reaction rate and dynamic or gated trapping [16] [17] [18] [19] [20] when the reaction is also modulated by an another independent reaction $T \leftrightarrow T^{\star}$ that switches the trap between an active and an inactive state.

Recently it has also been considered an evanescent Random Walk [21] [22] when the walkers have a finite lifetime.

Another extension of Smoluchowski's model is to consider mobile traps. Koza and Taitelbaum [13] have proposed a model of a mobile imperfect trap in the continuous space with the same diffusion coeficient for both species. Sánchez et al. [23] and Sánchez [24] have considered a mobile trap that diffuses but with no explicit diffusion model. In these works, the problem is considered in the trap reference system and in the laboratory system respectively.

In this article, we propose a generalized Continuous Time Random Walk for imperfect trapping with both species diffusing with different coeficients. We assume separable Random Walks and a time dependent reaction rate. In Section 2, we present a noninteracting two particles Random Walk to introduce the main magnitudes of the model. In Section 3, we include the reaction process in the model through an statiscally independent process. It is calculated in this section the local and global probability density for the time of reaction or the absorption probability density. In Section 4, an initial uniform distribution of the majority species is considered and we calculate the local and global reaction rate. It is verified that the global reaction rate coincides with the reaction rate for an immobile trap. The time dependent concentration of particles $A$ is also calculated. In Section 5, we present the results from the trap reference system. In this frame we recover some results of the immobile trap model. Finally in Section 6, we illustrate the results of our model with a one dimensional Random Walk. Discussion and conclusios follow.

\section{Two Particles Random Walk}

Let us consider a CTRW of two non-interacting particles on an homogeneous infinite lattice. The particles will be identified as $A$ and $T$ respectively. The 
position of each particle is given by an integer vector: $\quad \boldsymbol{x}$ for particle $A$ and $\boldsymbol{y}$ for particle $T$. Each particle makes a generalized, separable, statistically independent RW, with transitions rates $\lambda_{A} p_{A}\left(x-x^{\prime}\right)$ for a transition $\boldsymbol{x}^{\prime} \rightarrow \boldsymbol{x}$ of particle $A$ and $\lambda_{T} p_{T}\left(\boldsymbol{y}-\boldsymbol{y}^{\prime}\right)$ for a transition $\boldsymbol{y}^{\prime} \rightarrow \boldsymbol{y}$ of particle $T$ respectively. The sojourn probability of the walkers at a given configuration $(x, y)$ is

$$
\Phi_{0}(t)=\mathrm{e}^{-\lambda t}
$$

with $\lambda=\lambda_{A}+\lambda_{T}$. In this way the diffusion coeficients for a lattice parameter $a$ are respectively $D_{A}=a^{2} \lambda_{A} / 2 \mathrm{~d}$ and $D_{T}=a^{2} \lambda_{T} / 2 \mathrm{~d}$.

In turn the probability for a transition from configuration $\left(x^{\prime}, y^{\prime}\right)$ to configuration $(x, y)$ between $t$ and $t+\mathrm{d} t$ after a sojourn time $t$ is given by $\psi_{0}\left(\boldsymbol{x}-\boldsymbol{x}^{\prime}, \boldsymbol{y}-\boldsymbol{y}^{\prime} ; t\right) \mathrm{d} t$ with the probability density

$$
\psi_{0}\left(\boldsymbol{x}-\boldsymbol{x}^{\prime}, \boldsymbol{y}-\boldsymbol{y}^{\prime} ; t\right)=\left[\delta_{\boldsymbol{y}, \boldsymbol{y}^{\prime}} \lambda_{A} p_{A}\left(\boldsymbol{x}-\boldsymbol{x}^{\prime}\right)+\delta_{\boldsymbol{x}, \mathbf{x}^{\prime}} \lambda_{T} p_{T}\left(\boldsymbol{y}-\boldsymbol{y}^{\prime}\right)\right] \mathrm{e}^{-\lambda t}
$$

Here we are neglecting simultaneous transitions of both particles for being of second order in $d t$. This is the main magnitude in our model since all the results will be expressed in terms of this density.

Let $G_{0}\left(\boldsymbol{x}, \boldsymbol{y} ; t \mid \boldsymbol{x}_{0}, \boldsymbol{y}_{0}\right) \mathrm{d} t$ be the conditional probability of reaching configuration $(\boldsymbol{x}, \boldsymbol{y})$ between $t$ and $t+\mathrm{d} t$ given that the walkers started at the configuration $\left(\boldsymbol{x}_{0}, \boldsymbol{y}_{0}\right)$. The probability density $G_{0}$ satisfies the recursive relation

$$
\begin{aligned}
& G_{0}\left(\boldsymbol{x}, \boldsymbol{y} ; t \mid \boldsymbol{x}_{0}, \boldsymbol{y}_{0}\right) \\
& =\sum_{\boldsymbol{x}^{\prime}, \boldsymbol{y}^{\prime}} \psi_{0}\left(\boldsymbol{x}-\boldsymbol{x}^{\prime}, \boldsymbol{y}-\boldsymbol{y}^{\prime} ; t\right) \star G_{0}\left(\boldsymbol{x}^{\prime}, \boldsymbol{y}^{\prime} ; t \mid \boldsymbol{x}_{0}, \boldsymbol{y}_{0}\right)+\delta_{\boldsymbol{x}, \mathbf{x}^{\prime}} \delta_{\boldsymbol{y}, \boldsymbol{y}^{\prime}} \delta\left(t-0^{+}\right)
\end{aligned}
$$

since the walkers may reach the configuration $(x, y)$ by a transition from a previous configuration $\left(\boldsymbol{x}^{\prime}, \boldsymbol{y}^{\prime}\right)$ or $(\boldsymbol{x}, \boldsymbol{y})$ is the initial configuration, these being mutually exclusive events.

We are representing here by the symbol $\star$ the convolution product

$$
f(t) \star g(t)=\int_{0}^{\infty} \mathrm{d} t^{\prime} f\left(t-t^{\prime}\right) g\left(t^{\prime}\right)
$$

$G_{0}$ is the Green's function for the diffusion problem of two particles.

An analytic solution for the Green's function is obtained by taking Fourier transform in the spatial coordinates and Laplace transform in the time variable

$$
\hat{G}_{0}^{L}\left(\boldsymbol{k}_{A}, \boldsymbol{k}_{T} ; u \mid \boldsymbol{x}_{0}, \boldsymbol{y}_{0}\right)=\frac{\mathrm{e}^{i \boldsymbol{k}_{A} \cdot x_{0}} \mathrm{e}^{i \boldsymbol{k}_{T} \cdot y_{0}}}{1-\hat{\psi}_{0}^{L}\left(\boldsymbol{k}_{A}, \boldsymbol{k}_{T} ; u\right)}
$$

with

$$
\hat{\psi}_{0}^{L}\left(\boldsymbol{k}_{A}, \boldsymbol{k}_{T} ; u\right)=\frac{1}{u+\lambda}\left[\lambda_{A} p_{A}\left(\boldsymbol{k}_{A}\right)+\lambda_{T} p_{T}\left(\boldsymbol{k}_{T}\right)\right]
$$

Hereafter the superindex $L$ represents the Laplace transform in the temporal variable

$$
f^{L}(u)=\int_{0}^{\infty} \mathrm{d} t \mathrm{e}^{-u t} f(t)
$$


and the symbol $\wedge$ denotes the Fourier transform in spatial variables

$$
\hat{f}(\boldsymbol{k})=\sum_{\boldsymbol{x}} \mathrm{e}^{i \boldsymbol{k} \cdot \boldsymbol{x}} f(\boldsymbol{x})
$$

In this way the Green's function Fourier-Laplace $(\mathcal{F L})$ transform in $(5)$ is given in terms of the $\mathcal{F} \mathcal{L}$ transform of the transition probability density (2) only.

Jointly the conditional probability of a given configuraion at time $t$ of the particles is

$$
P_{0}\left(x, y ; t \mid x_{0}, y_{0}\right)=\Phi_{0}(t) \star G_{0}\left(x, y ; t \mid x_{0}, y_{0}\right)
$$

after reaching the confiugration $(x, y)$ at time $t^{\prime}$ the walkers must stay in these positions at least for a time $t-t^{\prime}$.

The marginal probability for the $A$ particle position

$$
P_{A}\left(x ; t \mid x_{0}\right)=\sum_{y} P_{0}\left(x, y ; t \mid x_{0}, y_{0}\right)
$$

is in the $\mathcal{F} \mathcal{L}$ transform representation

$$
\hat{P}_{A}^{L}\left(\boldsymbol{k}_{A} ; u \mid \boldsymbol{x}_{0}\right)=\Phi_{A}^{L}(u) \frac{\mathrm{e}^{i \boldsymbol{k}_{A} \cdot x_{0}}}{1-\hat{\psi}_{A}^{L}\left(\boldsymbol{k}_{A} ; u\right)}
$$

with

$$
\psi_{A}\left(x-x_{0} ; t\right)=\lambda_{A} p_{A}\left(x-x_{0}\right) \mathrm{e}^{-\lambda_{A} t}, \Phi_{A}(t)=\mathrm{e}^{-\lambda_{A} t}
$$

the transition probability density and sojourn probability for the $A$ walker. This result is consistent with the assumption of no interaction between the particles: particle $A$ diffuses with diffusion coeficient $D_{A}=a^{2} \lambda_{A} / 2 \mathrm{~d}$ on an infinite lattice of parameter $a$.

A similar result is obtained for the position marginal probability of particle $T$ position

$$
\hat{P}_{T}^{L}\left(\boldsymbol{k}_{A} ; u \mid \boldsymbol{y}_{0}\right)=\Phi_{T}^{L}(u) \frac{\mathrm{e}^{i \boldsymbol{k}_{T} \cdot y_{0}}}{1-\hat{\psi}_{T}^{L}\left(\boldsymbol{k}_{T} ; u\right)}
$$

with

$$
\psi_{T}\left(\boldsymbol{x}-\boldsymbol{x}_{0} ; t\right)=\lambda_{T} p_{T}\left(\boldsymbol{x}-\boldsymbol{x}_{0}\right) \mathrm{e}^{-\lambda_{T} t}, \Phi_{T}(t)=\mathrm{e}^{-\lambda_{T} t}
$$

If we have an uniform distribution of $A$ particles, $c_{0}$ in each lattice site, the particle concentration at site $\boldsymbol{X}$ at time $t$ is given by

$$
c_{A}(x ; t)=\sum_{x_{0}} P_{0}\left(x ; t \mid x_{0}\right) c_{0}
$$

and, replacing (5) in (9) and then in (14), we get in the $\mathcal{F} \mathcal{L}$ transform representation

$$
\hat{c}_{A}^{L}(\boldsymbol{k} ; u)=(2 \pi)^{d} \delta(\boldsymbol{k}) \frac{c_{0}}{u}
$$

with $d$ the dimension of space.

So it is verified that the uniform distribution is the equilibrium distribution for the noninteracting particles system. 


\section{The Reaction Process}

In this section we include in the model the possibility of the reaction $A+T \rightarrow T$ when both particles are at the same position in the lattice. In this case the particles may separate by one of them changing position or they may react with a time dependent reaction rate $\gamma(t)$. Assuming diffusion and reaction as statiscally independent processes, the sojourn probability of the walkers at the same position is [15]

$$
\Phi_{1}(t)=\exp \left[-\lambda t-\int_{0}^{t} \gamma(u) \mathrm{d} u\right]
$$

Concurrently the probability density for the time of separation of the particles is

$$
\psi_{1}\left(\boldsymbol{x}-\boldsymbol{x}^{\prime}, \boldsymbol{y}-\boldsymbol{y}^{\prime} ; t\right)=\psi_{0}\left(\boldsymbol{x}-\boldsymbol{x}^{\prime}, \boldsymbol{y}-\boldsymbol{y}^{\prime} ; t\right) \Xi_{r}(t)
$$

and the probability density for the time of reaction is

$$
\xi_{A}(t)=\xi_{r}(t) \mathrm{e}^{-\lambda t}
$$

with

$$
\Xi_{r}(t)=\exp \left[-\int_{0}^{t} \gamma(s) \mathrm{d} s\right]
$$

and

$$
\xi_{r}(t)=\gamma(t) \Xi_{r}(t)
$$

When particles $A$ and $T$ are not at the same position the state of the system can only change by one of the particles jumping to another position with probability density as given by (2).

We denote by $G_{r}\left(\boldsymbol{x}, \boldsymbol{y} ; t \mid \boldsymbol{x}_{0}, \boldsymbol{y}_{0}\right) \mathrm{d} t$ the Green's function for the diffusion of the particles when the reaction is allowed. It must satisfy the recursive relation

$$
\begin{aligned}
& G_{r}\left(\boldsymbol{x}, \boldsymbol{y} ; t \mid \boldsymbol{x}_{0}, \boldsymbol{y}_{0}\right) \\
& =\sum_{\boldsymbol{x}^{\prime}, \boldsymbol{y}^{\prime}} \psi\left(\boldsymbol{x}-\boldsymbol{x}^{\prime}, \boldsymbol{y}-\boldsymbol{y}^{\prime} ; t\right) \star G_{r}\left(\boldsymbol{x}^{\prime}, \boldsymbol{y}^{\prime} ; t \mid \boldsymbol{x}_{0}, \boldsymbol{y}_{0}\right)+\delta_{\boldsymbol{x}, \boldsymbol{x}^{\prime}} \delta_{\boldsymbol{y}, \boldsymbol{y}^{\prime}} \delta\left(t-0^{+}\right)
\end{aligned}
$$

This equation is similar to (3) but here

$$
\psi\left(x-x^{\prime}, y-y^{\prime} ; t\right)= \begin{cases}\psi_{1}\left(x-x^{\prime}, y-y^{\prime} ; t\right) & x^{\prime}=y^{\prime} \\ \psi_{0}\left(x-x^{\prime}, y-y^{\prime} ; t\right) & x^{\prime} \neq y^{\prime}\end{cases}
$$

We obtain an analytic solution of Equation (21) in the $\mathcal{F} \mathcal{L}$ transform representation by an extension of the local inhomogeneity method [15] [17] and making use of the identity [12]

$$
\delta_{x, \boldsymbol{y}}=\int_{0}^{2 \pi} \cdots \int_{0}^{2 \pi} \frac{\mathrm{d}^{d} \phi}{(2 \pi)^{d}} \exp [i(\boldsymbol{x}-\boldsymbol{y}) \cdot(\boldsymbol{\phi}-\boldsymbol{k})]
$$

with $d$ the dimension of the space.

The probability density for the time of both particles arriving at the same position $E\left(\boldsymbol{x}_{e} ; t \mid \boldsymbol{x}_{0}, \boldsymbol{y}_{0}\right)=G_{r}\left(\boldsymbol{x}_{e}, \boldsymbol{x}_{e} ; t \mid \boldsymbol{x}_{0}, \boldsymbol{y}_{0}\right)$ is in the $\mathcal{F} \mathcal{L}$ transform representation 


$$
\hat{E}_{r}^{L}\left(\boldsymbol{k} ; u \mid \boldsymbol{x}_{0}, \boldsymbol{y}_{0}\right)=\frac{I\left(\boldsymbol{k} ; u \mid \boldsymbol{x}_{0}-\boldsymbol{y}_{0}\right) \mathrm{e}^{\boldsymbol{k} \cdot \boldsymbol{y}_{0}}}{\Xi_{r}^{L}(u+\lambda)+\xi_{r}^{L}(u+\lambda) I(\boldsymbol{k} ; u \mid \mathbf{0})}
$$

with the definition

$$
I\left(\boldsymbol{k} ; u \mid \boldsymbol{x}_{0}-\boldsymbol{y}_{0}\right)=\frac{1}{(u+\lambda)} \int_{0}^{2 \pi} \cdots \int_{0}^{2 \pi} \frac{\mathrm{d}^{d} \boldsymbol{\phi}}{(2 \pi)^{d}} \frac{\exp \left[i \boldsymbol{\phi} \cdot\left(\boldsymbol{x}_{0}-\boldsymbol{y}_{0}\right)\right]}{1-\hat{\psi}_{0}^{L}(\boldsymbol{\phi}, \boldsymbol{k}-\boldsymbol{\phi} ; u)}
$$

while the Green's function is in the $\mathcal{F} \mathcal{L}$ transform representation

$$
\begin{aligned}
& \hat{G}_{r}^{L}\left(\boldsymbol{k}_{A}, \boldsymbol{k}_{T} ; u \mid \boldsymbol{x}_{0}, \boldsymbol{y}_{0}\right) \\
& =\hat{G}_{0}^{L}\left(\boldsymbol{k}_{A}, \boldsymbol{k}_{T} ; u \mid \boldsymbol{x}_{0}, \boldsymbol{y}_{0}\right)-\left[\frac{1}{1-\hat{\psi}_{0}^{L}\left(\boldsymbol{k}_{A}, \boldsymbol{k}_{T} ; u\right)}-1\right] \psi_{r}^{L}(u+\lambda) \hat{E}_{r}^{L}\left(\boldsymbol{k} ; u \mid \boldsymbol{x}_{0}, \boldsymbol{y}_{0}\right)
\end{aligned}
$$

More details of this calculation is given in Appendix A.

For the reaction to take place the walkers have to be at the same position. So the probability density for the time of reaction at a particular position $\boldsymbol{x}_{e}$ or local absorption probability density is given by the convolution product

$$
A_{l}\left(\boldsymbol{x}_{e} ; t \mid \boldsymbol{x}_{0}-\boldsymbol{y}_{0}\right)=\psi_{A}(t) \star E\left(\boldsymbol{x}_{e} ; t \mid \boldsymbol{x}_{0}-\boldsymbol{y}_{0}\right)
$$

since for the reaction to take place both particles have to meet at the same lattice site and react before they move away.

In the $\mathcal{F} \mathcal{L}$ representation we get from (18) and (24)

$$
\hat{A}_{l}^{L}\left(\boldsymbol{k} ; u \mid \boldsymbol{x}_{0}-\boldsymbol{y}_{0}\right)=\frac{I\left(\boldsymbol{k} ; u \mid \boldsymbol{x}_{0}-\boldsymbol{y}_{0}\right) \mathrm{e}^{\boldsymbol{i} \boldsymbol{k} \cdot \boldsymbol{y}_{0}}}{\frac{\Xi_{r}(u+\lambda)}{\xi_{r}(u+\lambda)}+I(\boldsymbol{k} ; u \mid 0)}
$$

With this result we may acknowledge Equation (26) as the $\mathcal{F} \mathcal{L}$ transform of

$$
\begin{aligned}
& G_{r}\left(\boldsymbol{x}, \boldsymbol{y} ; t \mid \boldsymbol{x}_{0}, \boldsymbol{y}_{0}\right) \\
& =G_{0}\left(\boldsymbol{x}, \boldsymbol{y} ; t \mid \boldsymbol{x}_{0}, \boldsymbol{y}_{0}\right)-\sum_{\boldsymbol{x}^{\prime}} G_{1}\left(\boldsymbol{x}, \boldsymbol{y} ; t \mid \boldsymbol{x}^{\prime}, \boldsymbol{x}^{\prime}\right) \star A_{t}\left(\boldsymbol{x}^{\prime} ; t \mid \boldsymbol{x}_{0}, \boldsymbol{y}_{0}\right)
\end{aligned}
$$

i.e. the Green's function for the reaction process is the Green's function of the two particles RW (3) minus the contribution of those realizations with a reaction at a previous time. Here

$$
G_{1}\left(x, y ; t \mid x^{\prime}, x^{\prime}\right)=G_{0}\left(x, y ; t \mid x_{0}, y_{0}\right)-\delta_{x, x^{\prime}} \delta_{y, y^{\prime}} \delta\left(t-0^{+}\right)
$$

is the probability density for the time of reaching the configuration $(x, y)$ after making at least one transition. For $\boldsymbol{x}=\boldsymbol{y}$ and $\boldsymbol{x}_{0}=\boldsymbol{y}_{0}$ this density is the equivalent of the probability density of the time of return to the origin for one particle random walk.

It is interesting to note that in the case of inmediate reaction upon encounter $\left(\gamma(t) \rightarrow \delta\left(t-0^{+}\right)\right)$

$$
\Xi_{r}(t)=\left\{\begin{array}{ll}
1 & t=0 \\
0 & t>0
\end{array}, \quad \xi_{r}(t)=\delta\left(t-0^{+}\right)\right.
$$

The probability density of the meeting time (in this case the first meeting due to inmediate reaction) independent of the lattice position is 


$$
\tilde{E}_{r}\left(t \mid \boldsymbol{x}_{0}, \boldsymbol{y}_{0}\right)=\sum_{\boldsymbol{x}_{e}} E\left(\vec{x}_{e} ; t \mid \boldsymbol{x}_{0}, \boldsymbol{y}_{0}\right)=\hat{E}_{r}\left(\boldsymbol{k}=0 ; t \mid \boldsymbol{x}_{0}, \boldsymbol{y}_{0}\right)
$$

Taking Laplace transform, evaluating Equation (24) at $\boldsymbol{k}=0$ and using (31) we get

$$
\tilde{E}_{r}^{L}\left(u \mid \boldsymbol{x}_{0}, \boldsymbol{y}_{0}\right)=\frac{I\left(0 ; u \mid \boldsymbol{x}_{0}-\boldsymbol{y}_{0}\right)}{I(0 ; u \mid 0)}
$$

$\tilde{E}_{r}^{L}\left(u \mid \boldsymbol{x}_{0}, \boldsymbol{y}_{0}\right)$ may be identified with Siegert's formula for the First Passage Time for the lattice position $\boldsymbol{y}_{0}$ of a walker that started a RW at $\boldsymbol{x}_{0}$ with transition probability density

$$
\psi_{E}\left(\boldsymbol{x}-\boldsymbol{x}^{\prime} ; t\right)=\left[\lambda_{A} p_{A}\left(\boldsymbol{x}-\boldsymbol{x}^{\prime}\right)+\lambda_{T} p_{T}\left(\boldsymbol{x}^{\prime}-\boldsymbol{x}\right)\right] \mathrm{e}^{-\lambda t}
$$

by recognizing the identity

$$
I\left(0 ; u \mid \boldsymbol{x}_{0}-\boldsymbol{y}_{0}\right)=G^{L}\left(\boldsymbol{x}_{0}-\boldsymbol{y}_{0} ; u\right)
$$

the Green's function of one particle Random Walk with transition probability density (34) on an infinite lattice [15]. Thus it is verified Smoluchowski's assumption that for the global reaction process we may consider one of the particles fixed on the lattice while the other diffuses with coeficient $D=D_{A}+D_{T}$ the sum of the individual diffusion coeficients.

The conditional probability of finding the walkers in configuration $(\boldsymbol{x}, \boldsymbol{y})$ at time $t$ assuming that they started at configuration $\left(\boldsymbol{x}_{0}, \boldsymbol{y}_{0}\right)$ is given by the convolution product

$$
P_{r}\left(\boldsymbol{x}, \boldsymbol{y} ; t \mid \boldsymbol{x}_{0}, \boldsymbol{y}_{0}\right)=\Phi(t) \star G_{r}\left(\boldsymbol{x}, \boldsymbol{y} ; t \mid \boldsymbol{x}_{0}, \boldsymbol{y}_{0}\right)
$$

with

$$
\Phi(t)= \begin{cases}\Phi_{1}(t) & \boldsymbol{x}=\boldsymbol{y} \\ \Phi_{0}(t) & \boldsymbol{x} \neq \boldsymbol{y}\end{cases}
$$

i.e. as in the case of (9), after reaching the configuration $(x, y)$ at time $t^{\prime}$ the walkers must stay in this configuration at least for a time $t-t^{\prime}$.

\section{Reaction Rate}

We consider in this section a distribution of particles $A$ with initial uniform concentration $c_{0}$ on the lattice. This is the equilibrium concentration in the absence of reaction for the diffusion model under consideration. We assume that the $A$ particles do not interact among them. Then the local reaction rate at $\boldsymbol{x}_{e}$ at time $t$ when $T$ starts at $y_{0}$; i.e. the number of particles that react at $x_{e}$ with $T$ between $t$ and $t+\mathrm{d} t$ is $\mathcal{R}_{l}\left(\boldsymbol{x}_{e} ; t \mid \boldsymbol{y}_{0}\right) \mathrm{d} t$ with the probability density

$$
\mathcal{R}_{1}\left(\boldsymbol{x}_{e} ; t \mid \boldsymbol{y}_{0}\right)=\sum_{x_{0}} A\left(\boldsymbol{x}_{e} ; t \mid \boldsymbol{y}_{0}\right) c_{0}
$$

In the $\mathcal{F} \mathcal{L}$ transform representation we get from (28)

$$
\hat{\mathcal{R}}_{1}^{L}\left(\boldsymbol{k} ; u \mid \boldsymbol{y}_{0}\right)=c_{0} \frac{\mathrm{e}^{i \boldsymbol{k} \cdot \boldsymbol{y}_{0}}}{u+\lambda_{T}\left(1-\hat{p}_{T}(\boldsymbol{k})\right)}\left[\frac{\Xi_{r}^{L}(u+\lambda)}{\xi_{r}^{L}(u+\lambda)}+I(\boldsymbol{k} ; u \mid 0)\right]^{-1}
$$


Here

$$
\hat{G}_{1}^{L}\left(\boldsymbol{k} ; u \mid \boldsymbol{y}_{0}\right)=\frac{\mathrm{e}^{i \boldsymbol{k} \cdot \boldsymbol{y}_{0}}}{u+\lambda_{T}\left(1-\hat{p}_{T}(\boldsymbol{k})\right)}
$$

is the $\mathcal{F} \mathcal{L}$ transform of Green's function of the isolated particle $T$ random walk.

The global reaction rate, defined as $\mathcal{R}_{g}(t)=\sum_{x} \mathcal{R}_{1}\left(x ; t \mid \boldsymbol{y}_{0}\right)$ is in the Laplace transform representation

$$
\mathcal{R}_{g}^{L}(u)=\frac{c_{0}}{u}\left[\frac{\Xi_{r}^{L}(u+\lambda)}{\xi_{r}^{L}(u+\lambda)}+\frac{1}{u}\right]^{-1}
$$

Note that the global reaction rate does not depend on the initial trap position as should be in a homogeneous lattice.

This reaction rate is coinciding with that of a set of walkers in the presence of a fixed trap when the walkers diffuses with the equivalent transition density (34).

In the presence of reaction the marginal probability of an $A$ particle position is

$$
P_{r, A}\left(x ; t \mid x_{0}, y_{0}\right)=\sum_{y} P_{r}\left(x, y ; t \mid x_{0}, y_{0}\right)
$$

with $P_{r}\left(\boldsymbol{x}, \boldsymbol{y} ; t \mid \boldsymbol{x}_{0}, \boldsymbol{y}_{0}\right)$ in (35). From this marginal probability we calculate the concentration of $A$ 's at a particular site

$$
C_{r}\left(\boldsymbol{x} ; t \mid \boldsymbol{y}_{0}\right)=\sum_{\boldsymbol{x}_{0}} P_{r, A}\left(\boldsymbol{x} ; t \mid \boldsymbol{x}_{0}, \boldsymbol{y}_{0}\right) c_{0}
$$

$\mathcal{F} \mathcal{L}$ transforming this equation, using (15) and (36) and noting that

$$
\Phi_{1}^{L}(u)-\Phi_{0}^{L}(u)=-\xi_{r}^{L}(u+\lambda) \Phi_{0}^{L}(u)
$$

we get

$$
\hat{c}_{r}{ }^{L}\left(\boldsymbol{k} ; u \mid \boldsymbol{y}_{0}\right)=(2 \pi)^{d} \delta(\boldsymbol{k}) \frac{C_{0}}{u}-\Phi_{0}(u) \hat{\mathcal{R}}_{1}{ }^{L}\left(\boldsymbol{k} ; u \mid \boldsymbol{y}_{0}\right)
$$

In the space-time representation this result corresponds to

$$
c\left(\boldsymbol{x} ; t \mid \boldsymbol{y}_{0}\right)=c_{0}-\Phi_{0}(t) \star \mathcal{R}_{1}\left(\boldsymbol{x} ; t \mid \boldsymbol{y}_{0}\right)
$$

the local concentration is the uniform initial concentration deducted the contribution of those $A$ particles that have reacted at a previous time $t^{\prime}<t$.

\section{Description from the Trap Reference System}

We present the previous results as described from the trap position. Let us start calculating the conditional probability of the relative position to the trap $T$ of an $A$ particle given by the coordinate $\mathbf{z}=\boldsymbol{x}-\boldsymbol{y}$. From (35) by the variable transformation method [25] [26]

$$
P_{Z}\left(\mathbf{z} ; t \mid \mathbf{z}_{0}\right)=\sum_{\boldsymbol{y}} P_{r}\left(\mathbf{z}+\boldsymbol{y}, \boldsymbol{y} ; t \mid \mathbf{z}_{0}+\boldsymbol{y}_{0}, \boldsymbol{y}_{0}\right)
$$

and in the $\mathcal{F} \mathcal{L}$ representation 


$$
\hat{P}_{Z}^{L}\left(\boldsymbol{k} ; u \mid \mathbf{z}_{0}\right)=\hat{P}_{0}^{L}\left(\boldsymbol{k},-\boldsymbol{k} ; u \mid \boldsymbol{x}_{0}, \boldsymbol{y}_{0}\right)-\frac{\Phi_{0}(u)}{1-\hat{\psi}_{0}^{L}(\boldsymbol{k},-\boldsymbol{k} ; u)} A_{g}^{L}(u)
$$

From this probablity we calculate the concentration of $A$ 's, that in the $\mathcal{F} \mathcal{L}$ representation is

$$
\hat{C}_{Z}^{L}(\boldsymbol{k} ; u)=(2 \pi)^{d} \delta(\boldsymbol{k}) \frac{C_{0}}{u}-\hat{P}_{Z}^{L}(\boldsymbol{k} ; u \mid 0) \mathcal{R}_{g}^{L}(u)
$$

Going back to the space time representation we may understand this result

$$
c_{Z}(\mathbf{z} ; t)=c_{0}-P_{Z}(\mathbf{z} ; t \mid 0) \star \mathcal{R}_{g}(t)
$$

in the following way: the concentration of $A$ 's at a given distance from the trap is given by the initial concentration subtracting the contribution of those realizations with a reaction at a previous time.

Note that the concentration as seen from the trap position is coinciding with the concentration for a fixed trap position as could be expected.

From this result we may conclude that the description of the reaction process from the trap position is comparable to the model of the trap at a fixed position on the lattice.

The main difference among the results at the reference systems appears in the $A$ 's concentration as given in (43) in the laboratory frame when compared with (47) in the trap frame.

\section{One Dimensional Random Walk}

We present here the results of our model when we consider an one dimensional Random Walk as schematically shown in Figure 1 . The probability density for the change of configuration is

$$
\begin{aligned}
\psi_{0}\left(\boldsymbol{x}-\boldsymbol{x}^{\prime}, \boldsymbol{y}-\boldsymbol{y}^{\prime} ; t\right)= & {\left[\delta_{y, y^{\prime}} \lambda_{A}\left(p_{d} \delta_{x, x^{\prime}+1}+p_{i} \delta_{x, x^{\prime}-1}\right)\right.} \\
& \left.+\delta_{x, x^{\prime}} \lambda_{T} p_{T}\left(\frac{1}{2} \delta_{y, y^{\prime}+1}+\frac{1}{2} \delta_{y, y^{\prime}-1}\right)\right] \mathrm{e}^{-\lambda t}
\end{aligned}
$$

so that particle $A$ makes a biased Random Walk. With this probability density the integral in (25) is

$$
I\left(k ; u \mid x_{0}-y_{0}\right)=\frac{R_{c}^{x_{0}-y_{0}} \zeta^{\left|x_{0}-y_{0}\right|}}{R_{p} R_{1}}
$$

where we have defined

$$
\begin{aligned}
& \lambda_{T} / 2 \quad \lambda_{T} / 2 \\
& \lambda_{A} p_{i} \quad \lambda_{A} p_{d} \\
& \stackrel{\circ}{\circ} \underset{\text { particle } \mathrm{T}}{\circ} \cdots \quad \circ \quad \circ \cdots
\end{aligned}
$$

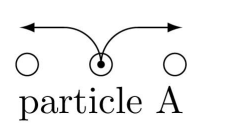

Figure 1. One dimensional Random Walk scheme. Transition rate for the trap $T$ is $\lambda_{T}$ and for particle $A$ is $\lambda_{A}$. Particle $T$ makes a symmetric Random Walk while particle $A$ makes a biased Random Walk: $p_{i}$ is the probability of jumping to the left and $p_{d}$ is the probability of jumping to the right. 


$$
\begin{array}{ll}
\Lambda_{d}=2 \lambda_{A} p_{d}+\lambda_{T} \mathrm{e}^{-i k} & \Lambda_{i}=2 \lambda_{A} p_{i}+\lambda_{T} \mathrm{e}^{i k} \\
R_{p}=\sqrt{\Lambda_{i} \Lambda_{d}} & R_{c}=\sqrt{\frac{\Lambda_{i}}{\Lambda_{d}}} \\
R_{1}=\sqrt{\left(\frac{u+\lambda}{R_{p}}\right)^{2}-1} & \zeta=\frac{u+\lambda}{R_{p}}-R_{1}
\end{array}
$$

For the reaction process we assume an exponential dynamics with a constant reaction rate $\gamma$

$$
\Xi_{r}(t)=\mathrm{e}^{-\gamma t}, \quad \xi_{r}(t)=\gamma \mathrm{e}^{-\gamma t}
$$

For this one dimensional model the local reaction rate in the $\mathcal{F} \mathcal{L}$ representation is

$$
\hat{\mathcal{R}}_{1}^{L}\left(k ; u \mid y_{0}\right)=\frac{\mathrm{e}^{i k y_{0}}}{u+\lambda_{T}(1-\cos k)} \frac{R_{p} R_{1}}{1+\frac{R_{p} R_{1}}{\gamma}} c_{0}
$$

and the $A$ 's concentration in the laboratory frame is

$$
\hat{c}_{r}^{L}\left(k ; u \mid y_{0}\right)=2 \pi \delta(k) \frac{c_{0}}{u}-\frac{c_{0}}{u+\lambda} \frac{\mathrm{e}^{i k y_{0}}}{u+\lambda_{T}(1-\cos k)} \frac{R_{p} R_{1}}{1+\frac{R_{p} R_{1}}{\gamma}}
$$

In turn the $A$ 's concentration in the trap frame is

$$
\hat{c}_{Z}^{L}(k ; u)=2 \pi \delta(k) \frac{c_{0}}{u}-\frac{c_{0}}{(u+\lambda)\left[1-\hat{\psi}_{A}^{L}(k, k ; u)\right]} \frac{\gamma}{u+\gamma}
$$

In Figure 2 we plot the normalized concentration $c / c_{0}$ in both reference frames: the laboratory frame and the trap frame as a function of the distance to the initial trap position, $x-y_{0}$, and as a function of the relative position to the trap, $z=x-y$, respectively. We include several curves with an adimensional time $\lambda t$ as a paramenter.

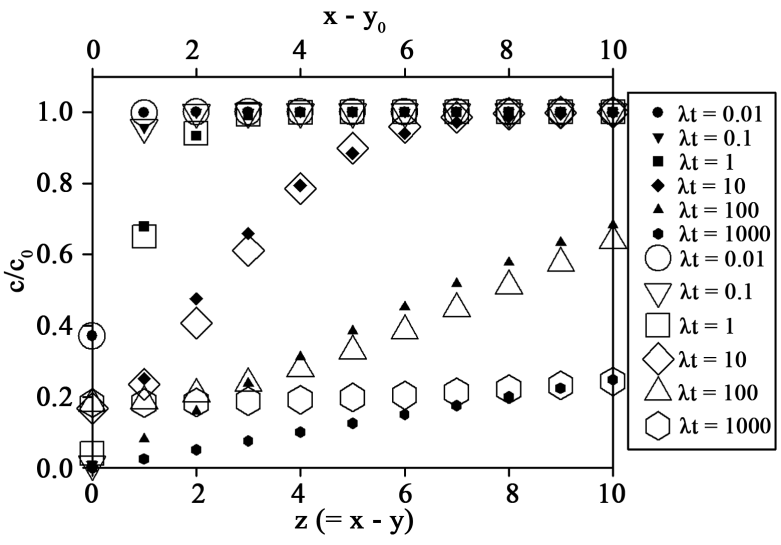

(a)

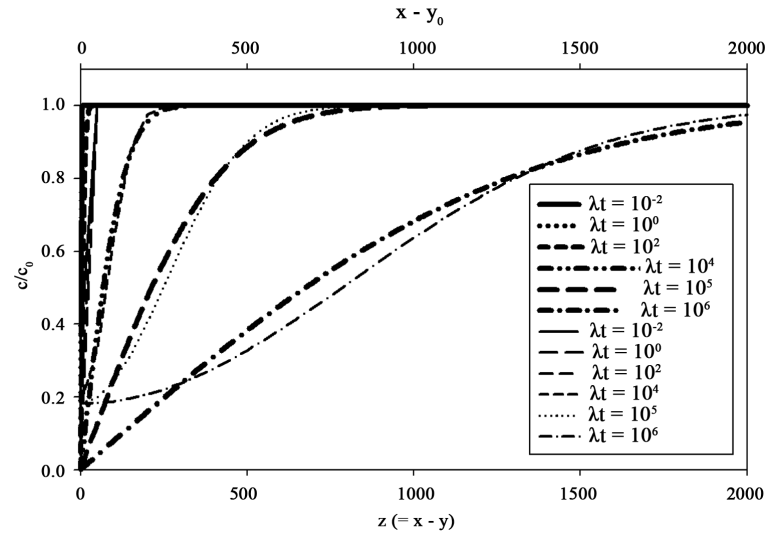

(b)

Figure 2. Majority species concentration in presence of a mobile imperfect trap vs. relative position to the trap, trap reference frame, (filled symbols in (a), thick lines in (b)) or vs. relative position to the initial position of the trap, laboratory reference frame, (hollowed symbols in (a), thin lines in (b)). It can be appreciated a deeper and narrower depletion zone around the trap in the trap reference frame. 
As can be seen there is a greater scavenging at the trap position when the concentration is calculated in the reference frame of the trap. A more extended scavenging region is observed around the initial trap position in the laboratory frame. This apparent disagreement can be understood if we take into account that in the laboratory frame we are considering an average realization of the trap Random Walk while in the trap frame we are just on the trap.

\section{Discussion and Conclusions}

We have presented a theoretical study of diffusion mediated reaction processes to extend previous treatments of imperfect trapping. Here both species, the minority and majority species, diffuse with arbitrary constants. We have calculated global and local absorption probability densities, reaction rates and concentration of the majority species in the laboratory and trap reference frames. All the results are expressed in terms of the relevant magnitudes of the model, the transition probability density between configurations of the system and the reaction dynamics. We have got analytical results in the Fourier Laplace transform representation.

Fixed trap absorption probability density and reaction rate are reobtained when we consider the global behaviour in the laboratory frame or in the trap frame of reference. The main difference between mobile and fixed trap models emerges when we consider the majority species concentration as can be appreciated in the one dimensional case of Section 6. In the trap frame, there is a deeper and spatially more reduced scavenging zone when compared with the concentration in the laboratory reference frame where the scavenging is not so deep but more extended spatially. This difference can be understood when we take into account that in the laboratory frame we are considering an average behaviour of the trap diffusion process, while in the trap frame we are considering a particular realization.

\section{Acknowledgements}

The authors thank SeCyT-UTN for partial support of this project.

\section{References}

[1] Rice, S.A. (1985) Diffusion-Limited Reactions. Elsevier, Amsterdam.

[2] Blumen, A., Klafter, J. and Zumofen, G. (1986) Optical Spectroscopy of Glasses. Reidel, Dordrecht.

[3] Goel, N.S. and Richter-Dyn, N. (1974) Stochastic Models in Biology. Academic, New York.

[4] Alberts, B., Bray, D., Lewis, J., Raff, M., Roberts, K. and Watson, J. (1992) Biologa Molecular de la Célula. Omega, Barcelona.

[5] Viswanathan, G.N., Buldyrev, S.V., Havlin, S., da Luz, M.G.E., Raposo, E.P. and Stanley, H.E. (1999) Optimizing the Success of Random Searches. Nature, 401, 911-914. https://doi.org/10.1038/44831

[6] von Smoluchowski, R. (1917) Versucheiner Mathematischen Theorie der Koagula- 
tions Kinetic Kolloider Lousungen. Zeitschrift für Physikalische Chemie, 92.

[7] Scher, H. and Lax, M. (1973) Stochastic Transport in a Disordered Solid. I. Theory. Phys. Rev. B, 7. https://doi.org/10.1103/PhysRevB.7.4491

[8] Scher, H. and Lax, M. (1973) Stochastic Transport in a Disordered Solid. II. Impurity Conduction, Physical Review B, 7, 4491.

[9] Bendler, J.T. and Shlesinger, M.F. (1985) The Wonderful World of Stochastics. Elsevier, Amsterdam.

[10] Collins, F.C. and Kimball, G.E. (1949) Diffusion-Controlled Reaction Rates. Journal of Colloid Science, 4, 425-437. https://doi.org/10.1016/0095-8522(49)90023-9

[11] Noyes, R.M. (1954) A Treatment of Chemical Kinetics with Special Applicability to Diffusion Controlled Reactions. The Journal of Chemical Physics, 22, 1349-1359. https://doi.org/10.1063/1.1740394

[12] Aslangul, C. (1999) Mutual Annihilation of Two Diffusing Particles in One- and Two-Dimensional Lattices. Journal of Statistical Physics, 94, 219-240.

[13] Koza, Z. and Taitelbaum (1998) Spatiotemporal Properties of Diffusive Systems with a Mobile Imperfect Trap. Physical Review E, 57, 237-243. https://doi.org/10.1103/PhysRevE.57.237

[14] Condat, C.A. (1989) Solution to the Glarum Model with a Finite Relaxation Rate. Zeitschrift für Physik B Condensed Matter, 77, 313-320.

[15] Ré, M. and Budde, C.E. (2000) Diffusion-Mediated Reactions with a Time Dependent Absorption Rate. Physical Review E, 61, 1110-1120.

[16] Budde, C.E., Cáceres, M.O. and Ré, M.A. (1995) Transient Behaviour in the Absorption in the Presence of a Non-Markovian Probability Distribution Dynamic Trap. Europhysics Letters, 32, 205.

[17] Cáceres, M.O., Budde, C.E. and Ré, M.A. (1995) Theory of the Absorption Probability Density of Diffusing Particles in the Presence of a Dynamic Trap. Physical Review $E, 52,3462$.

[18] Ré, M.A., Budde, C.E. and Cáceres, M.O. (1996) Survival Probability in the Presence of a Dynamic Trap. Physical Review E, 54, 4427.

[19] Spouge, J.L., Szabo, A. and Weiss, G.H. (1996) Single-Particle Survival in Gated Trapping. Physical Review E, 54, 2248. https://doi.org/10.1103/PhysRevE.54.2248

[20] Bressloff, P.C., Karamched, B.R., Lawley, S.D. and Levien, E. (2017) Diffusive Transport in the Presence of Stochastically Gated Absorption. Physical Review E, 96, Article ID: 022102. https://doi.org/10.1103/PhysRevE.96.022102

[21] Yuste, S.B., Abad, E. and Linenberg, K. (2013) Exploration and Trapping of Mortal Random Walkers. Physical Review Letters, 110, Article ID: 220603. https://doi.org/10.1103/PhysRevLett.110.220603

[22] Ré, M. and Bustos, N.C. (2015) Reaction Rate in an Evanescent Random Walkers System. Papers in Physics, 7, Article ID: 070003. https://doi.org/10.4279/pip.070003

[23] Sánchez, A.D., Rodrguez, M.A. and Wio, H.S. (1998) Results in Trapping Reactions for Mobile Particles and a Single Trap. Physical Review E, 57, 6390.

[24] Sánchez, A.D. (1999) Trapping Reactions for Mobile Particles and a Trap in the Laboratory Frame. Physical Review E, 59, 5021. https://doi.org/10.1103/PhysRevE.59.5021

[25] Papoulis, A. (1991) Probability, Random Variables and Stochastic Processes. McGraw-Hill, Singapore.

[26] Gillespie, D.T. (1982) A Theorem for Physicist in the Theory of Random Variables. American Journal of Physics, 51, 520-533. 


\section{Appendix A: Local Inhomogeneity Method}

Starting with the recursive relation (21) in the Laplace representation we work out the sum $\sum_{x^{\prime}, y^{\prime}} G_{0}^{L}\left(\boldsymbol{x}, \boldsymbol{y} ; u \mid \boldsymbol{x}^{\prime}, \boldsymbol{y}^{\prime}\right) G_{r}^{L}\left(\boldsymbol{x}^{\prime}, \boldsymbol{y}^{\prime} ; u \mid \boldsymbol{x}_{0}, \boldsymbol{y}_{0}\right)$. Noting that in the Laplace representation

$$
\begin{aligned}
& \psi_{1}^{L}\left(\boldsymbol{x}-\boldsymbol{x}^{\prime}, \boldsymbol{y}-\boldsymbol{y}^{\prime} ; u\right)-\psi_{0}^{L}\left(\boldsymbol{x}-\boldsymbol{x}^{\prime}, \boldsymbol{y}-\boldsymbol{y}^{\prime} ; u\right) \\
& =-\xi_{r}^{L}(u+\lambda) \psi_{0}^{L}\left(\boldsymbol{x}-\boldsymbol{x}^{\prime}, \boldsymbol{y}-\boldsymbol{y}^{\prime} ; u\right)
\end{aligned}
$$

and making use of the recursive relation (3) we get

$$
\begin{aligned}
& G_{r}^{L}\left(\boldsymbol{x}, \boldsymbol{y} ; u \mid \boldsymbol{x}_{0}, \boldsymbol{y}_{0}\right) \\
& =G_{0}^{L}\left(\boldsymbol{x}, \boldsymbol{y} ; u \mid \boldsymbol{x}_{0}, \boldsymbol{y}_{0}\right)-\xi_{r}(u+\lambda) \sum_{\chi^{\prime}, y^{\prime}} \delta_{x^{\prime}, \boldsymbol{y}^{\prime}}\left[G_{0}^{L}\left(\boldsymbol{x}, \boldsymbol{y} ; u \mid \boldsymbol{x}^{\prime}, \boldsymbol{y}^{\prime}\right)-\delta_{\boldsymbol{x}, x^{\prime}} \delta_{\boldsymbol{y}, \boldsymbol{y}^{\prime}}\right] \\
& \times G_{r}^{L}\left(\boldsymbol{x}^{\prime}, \boldsymbol{y}^{\prime} ; u \mid \boldsymbol{x}_{0}, \boldsymbol{y}_{0}\right)
\end{aligned}
$$

It should be noted that in the right hand side it appears $G_{r}^{L}\left(\boldsymbol{x}^{\prime}, \boldsymbol{x}^{\prime} ; u \mid \boldsymbol{x}_{0}, \boldsymbol{y}_{0}\right)=E_{r}^{L}\left(\boldsymbol{x}^{\prime} ; u \mid \boldsymbol{x}_{0}, \boldsymbol{y}_{0}\right)$ as defined in (24). Then we evaluate (56) at $\boldsymbol{x}=\boldsymbol{y}$ and take Fourier transform obtaining (24). For this calculation use have been made of identity (23) and definition (25). 